trol the two thicknesses. These have almost universally consisted of combinations of layers of GaAs and of the higher bandgap and lower refractive index mixed semiconductor, (GaAl)As. The higher bandgap can be used to introduce an additional potential barrier which will confine injected carriers to a layer of almost any desired thickness and the lower refractive index provides a similar although more limited function in respect of guiding the light. The success of this approach, particularly as regards carrier confinement, has relied very strongly on the almost ideal nature of the $\mathrm{GaAs} /(\mathrm{GaAl})$ As single crystal interface, which is a result of the close lattice match of the two materials. This good match is a lucky accident of nature and to obtain comparable behaviour with other semiconductor materials the more technologically demanding four element mixed semiconductors would have to be used.

The progress of heterostructure lasers over the last four years has been from the two layer so called 'single heterostructure' (SH) to the three layer 'double heterostructure' (DH) and finally this year to the five layer 'double confinement heterostructure' (DCH). RCA, the Ioffe Institute and Standard Telecommunication Laboratories have shared the honours with, in each case, Bell Telephone Laboratories for developing these respective layouts.

In the first two types of device both light and injected carriers are confined to a single GaAs layer bounded either by a $\mathrm{p}-\mathrm{n}$ junction on one side and a heterojunction on the other (SH), or by two heterojunctions (DH). In both cases the limit on the thinness of the GaAs layer is determined by the value at which optical guiding starts to deteriorate, the respective figures being about 2 and $0.15 \mu \mathrm{m}$. In the third case only the injected carriers are confined to the centre GaAs layer, whose thickness can be reduced almost without limit. The present lowest figure being quoted is $0.04 \mu \mathrm{m}$ by Standard Telecommunication Laboratories. The centre layer is surrounded by two layers of intermediate AlAs content giving a combined thickness adequate for optical guiding (not less than about $0.2 \mu \mathrm{m}$ ) and the two outermost layers of higher AlAs content provide the optical confinement barriers.

In the SH and DH devices the threshold current density is almost directly proportional to the active layer thickness, best figures at $300{ }^{\circ} \mathrm{C}$ being about 7 and $1 \mathrm{kA} \mathrm{cm}^{-2}$ respectively. In the $\mathrm{DCH}$ lasers the threshold current density does not increase in exact proportion to the reduction of the active layer thickness since the same optical power has still to be supplied to the guided light. However, a considerable current density is necessary to convert the GaAs from a lossy to a transparent state before any appreciable gain is produced and this current is saved by reducing the proportion of the light guide which consists of $\mathrm{GaAs}$ and has to be pumped. At present the lowest threshold current densities obtained in DCH devices are around $500 \mathrm{~A} \mathrm{~cm}^{-2}$ but further improvements are likely.

When threshold current densities are below about $1.5 \mathrm{kA} \mathrm{cm}^{-2}$ it is possible to obtain CW operation at room temperature with $0.5 \mathrm{~A}$ current from devices about $0.3 \mathrm{~mm}$ long and up to $0.1 \mathrm{~mm}$ wide by simply mounting them on a copper heat sink. A laser as wide as this is not needed in many applications and by making much narrower devices it is possible to get much better control of the laser resonances and approach single mode (truly coherent) operation. At present lateral confinement of the light (in the plane of the layers) is achieved by various systems which limit the current flow, and therefore the region of gain, to a narrow stripe in the active layer. This method operates satisfactorily down to a width of about $10 \mu \mathrm{m}$. Such devices can give single mode operation if they are not driven too hard. Workers at Toshiba have measured frequency stability in several devices better than $\pm 100 \mathrm{MHz}$. When such results can be reproduced at will injection lasers will start having applications for holographic work.

$\mathrm{GaAs} /(\mathrm{GaAl})$ As injection lasers are in many respects particularly suitable for use as sources in optical systems of communication by single mode fibre. Their wavelength of around $0.9 \mu \mathrm{m}$ lies at the point where the attenuation of glass reaches a low value between scattering losses at short wavelengths and molecular absorption losses at longer wavelengths. Their size is compatible with the small diameter (about $3 \mu \mathrm{m}$ ) of the core of the fibre. Due to their short length and the short spontaneous recombination time of the injected carriers (about $3 \mathrm{~ns}$ ) they can be modulated directly at a high rate. Telefunken has achieved modulation rates of $2 \cdot 3$ gigabits per second by biasing at threshold.

Finally injection lasers are very suitable for integrating with the large variety of other optical components which can be made in the GaAs/(GaAl)As system. The one characteristic which remains unsatisfactory is the operating life of these lasers. However, great progress is being made at the moment. Both Standard Telecommunication Laboratories and Nippon Electric Company have this year operated devices $\mathrm{CW}$ for up to $4000 \mathrm{~h}$ and found no fundamental process which limits the life. When this figure is improved by another order of magni- tude the versatility and excellent performance of the semiconductor lasers should quickly open up a large range of useful applications

\section{Nonlinear mixing in vapours}

By Dr D C HANNA, MInstP

It is now more than ten years since the first experiment which demonstrated that the generation of harmonics, a commonplace phenomenon at audio and radiofrequencies, could also be made to occur at optical frequencies. Just as in the low frequency case, an element with a nonlinear response is needed to achieve this and at optical frequencies this is provided by the nonlinear dependence of polarization on the applied field. This is expressed by the equation

$$
P=\varepsilon_{0} \chi_{1} E+\varepsilon_{0} \chi_{2} E^{2}+\varepsilon_{0} \chi_{3} E^{3}+\text { etc. }
$$

The first term, containing the first order susceptibility $\chi_{1}$, gives rise to the familiar linear optical property of an index of refraction. Although $\chi_{2}$ is small, the optical fields produced by lasers can be so great that effects due to the term $\varepsilon_{0} \chi_{2} E^{2}$ are no longer insignificant and one can build practical devices which exploit this.

The most important effects are sum frequency generation, $\omega_{1}+\omega_{2}=\omega_{3}$, (of which second harmonic generation is a special case, $\omega+\omega=2 \omega)$ and difference frequency generation, $\omega_{1}-\omega_{2}=\omega_{3}$. Thus by difference frequency generation from two visible lasers close in frequency one can produce a beam of coherent radiation at infrared wavelengths for which lasers are not available. Similarly sum frequency generation of visible lasers can extend the range of coherent sources into the ultraviolet. By using frequencytunable dye lasers to provide the initial frequencies $\omega_{1}$ and $\omega_{2}$, the generated infrared or ultraviolet radiation has also been made tunable. Wide applications in spectroscopy are foreseen and are already being demonstrated for these tunable infrared and ultraviolet sources. However, there is great interest in extending further into the infrared and further into the ultraviolet. In both of these directions important advances have been made in the last year by exploiting effects due to the higher order nonlinear term $\varepsilon_{0} \chi_{3} E^{3}$.

A symmetry argument shows that $\chi_{2}$ is nonzero only for materials lacking inversion symmetry. This was at first seen as eliminating isotropic materials such as gases and liquids for efficient mixing or harmonic generation and the number of useful nonlinear materials was therefore rather restricted. Also, since nonzero $\chi_{2}$ is found only in crystalline (and therefore solid) materials, the useful range of these materials is limited both in the UV and IR by the fundamental absorption behaviour of solids. These 
limits are avoided in gases as is the problem of optically induced damage which is found to plague solids. But in gases one must rely on the nonlinear term $\varepsilon_{0} \chi_{3} E^{3}$ and generally terms of higher order of nonlinearity are smaller. The term $\varepsilon_{0} \chi_{3} E^{3}$ in a gas might therefore be expected to be much less than the term $\varepsilon_{0} \chi_{2} E^{2}$ for a typical solid, the more so as the atomic density is much less in a gas.

However it is found that the expressions for $\chi_{3}$ contain resonance denominators such that $\chi_{3}$ becomes very large for frequencies which are close to real transition frequencies between energy levels of the gas atoms. Under these conditions effects due to $\chi_{3}$ in gases can become comparable to effects due to $\chi_{2}$ in solids. The important effects which result from $\chi_{3}$ are sum frequency generation $\omega_{1}+\omega_{2}+\omega_{3}=\omega_{4}$ (of which third harmonic generation is the special case $\omega+\omega+\omega=3 \omega)$; and difference frequency generation, $\omega_{1}-\omega_{2}-\omega_{3}=\omega_{4}$. Using these effects, and starting with visible lasers, the generated IR and UV wavelengths have so far been extended to $25 \mu \mathrm{m}$ and $89 \mathrm{~nm}$ and further extensions can be expected.

Both the difference frequency generation and the third harmonic generation schemes at first have used alkali metal vapours as the nonlinear medium. Third harmonic generation has now been reported in mixtures of inert gases (Xe and $\mathrm{Ar}$ ) with conversion efficiency from 354 to $118 \mathrm{~nm}$ of a few percent with powers of several hundred kilowatts at $118 \mathrm{~nm}$. A mixture of gases is used in order to adjust the refractive indices of the fundamental wave and the third harmonic wave to be equal. Similarly in difference frequency generation one requires this refractive index matching and although this can be achieved with a single vapour (such as potassium) the best result, which includes a continuous tuning range from 2 to $25 \mu \mathrm{m}$ with powers ranging from $0.1 \mathrm{~W}$ to $0.1 \mathrm{~mW}$, has been obtained with a mixture of sodium and potassium vapour. It is the potassium vapour which provides the nonlinear behaviour, the sodium being used merely to adjust the refractive index.

The scheme of potassium energy levels and mixing frequencies is shown in figure 9. A blue dye laser (frequency $\omega_{1}$ ) is tuned to be close to resonance with the $4 s-5 p$ transition. This leads to powerful generation of stimulated resonance $\mathrm{Ra}$ man scattered radiation of frequency $\omega_{2}$. Another dye laser of frequency $\omega_{3}$ provides a third frequency and by mixing these three frequencies, a fourth frequency, $\omega_{4}=\omega_{1}-\omega_{2}-\omega_{3}$, is generated in the infrared. Tuning of the dye laser $\omega_{3}$ leads to tuning of the infrared frequency $\omega_{4}$. In addition to the exciting feature of an extremely wide tuning range ( 2 to $25 \mu \mathrm{m}$ so far) the infrared

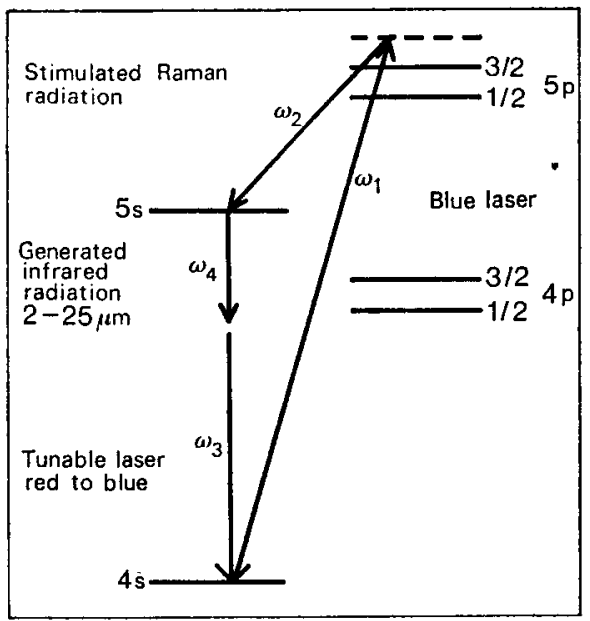

Figure 9 The scheme of potassium energy levels and mixing frequencies

linewidth even in these first experiments has been only about $0.2 \mathrm{~cm}^{-1}$.

These are as yet early days for nonlinear mixing in vapours and future improvements which can be expected are; wider tuning ranges, narrower linewidths and much greater powers since dye laser powers are being steadily improved. For the ultraviolet generation in vapours, recent calculations and experiments by Harris show that much higher order nonlinearities in vapours can be large and can therefore be used to extend coherent sources into the soft $x$ ray region

\section{FURTHER READING}

Harris S E 1973 Phys. Rev. Lett. 31 341-4

Kung A H, Young J F and Harris S E 1973 Appl. Phys. Lett. 22 301-2

Miles R B and Harris S E IEEE J. Quantum Electronics, QE-9 470-84

Sorokin P P, Wynne J J and Lankard J R Appl. Phys. Lett. 22 342-4

Smith R C 1970 Physics Bulletin 21 341-4

Wynne J J, Sorokin P P and Lankard J R 1973 Proc. Conf. on Laser Spectroscopy, Vail, Colorado, 1973

\section{High voltage electron microscopy}

\section{By Dr C J HUMPHREYS, MInstP}

The recent considerable investment in high voltage electron microscopy (HVEM) notably by the USA, France, Japan and the UK, has undoubtedly produced some scientific dividends in the last 12 months. Advances have been made in a wide range of disciplines, and the siting of the third international conference on HVEM in Oxford this year was to some extent in recognition of the prominence of the UK in this field.

A standard accelerating voltage in HVEM is $1 \mathrm{MV}$, and $1 \mathrm{MeV}$ electrons have sufficient energy to displace atoms from their lattice sites in many crystalline materials. One topical application of this is the technologically important problem of void formation in nuclear reactor materials. This problem may be

simulated in HVEM by using incident fast electrons, rather than neutrons, to produce the damage and to image simultaneously the growth of the voids. Considerable progress, of both scientific and economic value, in this area has been made this year at Harwell, the CEGB, Oak Ridge and various universities.

When a crystal is set at a Bragg reflecting position a high diffracted intensity is normally expected due to "constructive interference'. One of the unexpected discoveries made using HVEM is that for a particular incident electron energy the diffracted intensity may minimize rather than maximize. The electron accelerating voltage corresponding to this particular energy is known as the critical voltage, which is a function of the material, Bragg reflection and temperature. The precision with which the critical voltage effect may be used to determine lattice potentials, electron densities and solid state bonding effects has been established by Hewat at Oxford this year, who has shown that in many cases this HVEM method will be more accurate and also considerably faster than $\mathrm{x}$ ray methods.

Jones at Birmingham is applying this method to determine bonding effects in hexagonal metals having large deviations from the 'ideal' $c / a$ ratio, and Butler at Imperial College and Thomas in America have carried out some preliminary experiments indicating that the effect is sensitive to both long range and short range ordering in alloys.

The increased penetration in HVEM, relative to conventional $100 \mathrm{kV}$ electron microscopy, enables thicker specimens to be studied, the properties of which are less influenced by surface effects and hence more similar to the properties of bulk materials. This enables meaningful studies to be made of many materials for the first time. For example this year the British Steel Corporation has used HVEM to characterize various steels, some of the results being fed back into the manufacturing process. In addition a wide range of dynamic in situ experiments is being performed with the HVEM using specially constructed straining, heating, cooling, etc, specimen stages. Thus dislocation generation and multiplication are being studied in situ at Birmingham and Oxford, the deformation of superplastics at the NPL, solid state transformations at Imperial College, and so on.

The increased penetration available in HVEM may be used not only to study relatively thick specimens but also to study somewhat thinner specimens surrounded by a controlled liquid or gaseous environment contained in an environmental cell within the microscope. Swann and coworkers at Imperial Col- 